Original Contribution

\title{
REPORTING OF ELECTRICITY THROUGH THE INTRODUCTION OF HAND PORTABLE TERMINALS
}

\author{
J. Angelova* \\ Department of Economics, Industrial Engineering and Management, Technical University of Sofia, \\ Bulgaria
}

\begin{abstract}
The aim of the study is to analyze the reporting of electricity on paper and by introducing handheld portable terminals. It has been used a method of introducing handheld portable terminals. Outlined are the relevant results, and that draws conclusions from the study. In the conclusion, the author offers options to avoid shortcomings in the data sampling from commercial measuring and recording them on paper.
\end{abstract}

Key words: electricity, portable terminals, commercial metering.

\section{INTRDUDCTION}

Energy security is a key component of the overall security of the European Union / EU /. Narrowness of the world's energy resources in the medium term and the EU's dependence on energy imports pose a number of questions relating to the need to ensure supply and finding alternative sources.

A number of strategic documents focus the efforts of the EU institutions on issues of energy security. One of them is the European energy security strategy adopted by the European Commission in May 2014. It states that EU energy security should be considered in the context of growing demand for energy worldwide, which is expected to increase by $27 \%$ by 2030 .

In this regard there are several main pillars through which the measures will benefit all Member States of the EU, and whilst into account all national decisions. Some of the most important measures are:

- Strengthening the mechanisms to respond to emergencies and solidarity mechanisms, including coordination of risk assessments and action plans in emergencies and protection of strategic infrastructure;

- Reducing the demand for energy;

○ Building a well-functioning and fully integrated internal market;

Correspondence to: Assoc.Prof. Jordanka Angelova, PhD, TU-Sofia, e-mail: jsa@tusofia.bg
- Diversification of external supplies and related infrastructure and others.

There is a need for closer coordination of national energy policies to find udebitelen response to the challenge of energy security. Also there are additional conditions required to ensure better interaction between energy and foreign policy objectives and speaks with one voice to partners of the EU.

\section{Rules for measuring the amount of electricity}

The adopted rules to measure the amount of electricity [1] define crucial aspects such as: 1. Principles, ways and places to measure the amount of active and reactive power;

2.Requirements for accuracy of measurement, verification of measurement systems and related communications;

3 . The creation, maintenance and access to the database of the measuring system;

4. Registration of the commercial measurement of quantities of active and reactive power; 5. The rights and obligations of the parties to the transactions with electricity, the operator of the electricity system and distribution network operators related to the measurement of the amount of electrical energy.

According to the adopted rules, all electricity for commercial and control measurement reporting and recording kilowatt hours $(\mathrm{kWh})$ at the measuring of active and / or capacitive / inductive reactive power. Quantities of active 
and reactive power at any point of measurement are recorded at least once a month, and the period can not be longer than 31 days.

In terms of technical requirements, metrological characteristics and specifications of the measuring system, they are determined by the voltage level at the measuring point and the amount of electricity that will be measured.

The point of connection to the electricity, respectively the distribution network shall be determined in the Accession Treaty in accordance with the Regulation for connecting producers and consumers of electric power transmission and distribution electrical grids of art. 116, para. 7 of the Energy Act [2].

The produced electricity and the electricity used by consumers is measured by means of commercial metering - owned distribution company.

- The distribution company is obliged to deliver, install and maintain the commercial metering / electricity / and related equipment for recording and transmission of data in cases when measured:

- The energy used by energy users for business purposes, connected to the distribution network of medium and low voltage;

- The energy used by household consumers connected to distribution networks Low Voltage;

- The energy entering the electrical installations of eligible consumers connected to the electricity grid;

- Thew Energy producer connected to the distribution network. [3] Delivery, installation and maintenance of the commercial metering devices and related equipment for recording and transmission of data in places of exchange of energy between neighboring distribution companies shall be governed by contract concluded between them.

The distribution company is obliged to provide the country which buys electricity, access for visual control the electric meter readings. And the consumers in turn are obliged to provide officials from the distribution company, unhindered access to all parts of the measuring system for their installation, inspection and reporting the quantities of electricity. All costs except the cost of measuring instruments themselves are borne by the consumer.
Determining the location of measurements for the consumed electricity.

Upon receipt of electricity from producers to the distribution network, the place of measurements is the high voltage side of the step-up transformers.

In giving power of electricity to the power grid position measurement is part of the medium voltage step-down transformer and side $110 \mathrm{kV}$, which is owned by the distribution company. It is measured the submitted net amount of electricity.

When giving electricity from the grid, respectively the distribution network, to connected consumers, the place of measurement is the country with higher voltage lowering transformer user (if any transformation) or in the connection of users to the electricity, respectively the distribution network.

Technological costs of energy in a facility (equipment) are at the expense of its owner.

Upon submission of electricity from the transmission or distribution company to a manufacturer, the place of measurements is at the high voltage side.

In the interconnection point the measurements are organized by the transmission company in the border substation (system) as recommended by the UCTE and bilateral supply agreements (exchange) of electricity.

The agreed upon rules allow when the place of measurements does not coincide with the point of connection, metering data may if necessary be adjusted. In this case, transmission or the distribution company and the country whose energy is measured agree on compensation. The agreed procedure for determining the amount of compensation is included in the register of the measurement system.

An analysis of reporting of the utilized electricity via handheld portable terminals.

The used electricity is according to set schedules and region.

Place of work are switchboards of household and industrial consumers whose electricity is measured by meters for directly or indirectly measurements the level of low voltage on the territory served by the "CEZ Distribution Bulgaria", to set a timetable to specify measuring points. 
The overall award of work is done by worksites of direction "Reporting data."

\section{Volume and method of performed works}

The work done is a monthly reading of assigned meters in separate territorial regions of the territory served by the "CEZ Distribution Bulgaria" and examined for damage and irregularities measurement group (electrometer, tariff switch, instrument transformers, panel and others. ).

Electrical energy meters are grouped into units. Reporting unit is the smallest indivisible group of instrumentation that is loaded at a terminal. In a reporting unit includes meters in certain distinct region of the territory served by the worksite.

All units are divided into 15 periods, in a month the person responsilbe will read five of those periods in the second month next five and in the third month - the other five periods, i.e. all units will report be within three months and then the cycle begins again. Reporting units that are included in the ten periods are not awarded in a given month, but they are reported by other reporting on the same principle, ie within three months, receiving rotation between three groups of reporting. At the beginning of the year group I started with reporting the reporting agents of the first period; Group II - starting from 6 period; Group III - beginning of period 11.

Quantities of electricity awarded for reporting within one calendar month is divided into five (5), the period of 4 (four) business days for everyone.

In order to avoid increasing the days between two monthly report due to accumulation of weekends and holidays, the schedule can be altered to include work during weekends and holidays. within the statutory deadline.

Meters in one reporting unit must be reported within one reporting period, the reporting period for each meter can not be greater than the statutory definitions.

The main part of the meters are mounted on standard height - over $3 \mathrm{~m}$., And a few of them at a height over $3 \mathrm{~m}$. In the process of reading into account all settlements, neighborhoods, recreation areas, separate objects and others that are powered by electricity and included organizationally in the territory of a Regional Unit.

Service reporting process of settlement of a particular work area is not connected with the administrative division of the settlements on the territory of Bulgaria.

Reading of meters is carried on where they are installed the meters of customers. The company organizes the reading of meters, using available database client system My Avis - client information to the award of the account of the reporting entity.

It is important to note that actual reporting is electric meter whose readings are displayed on the meter at the time of the report and immediately thereafter entered in the terminal.

\section{Approach and stages of consideration of the consumed electricity}

The reading of electricity meters and other related activities are carried out in accordance with current legislation. The activities are performed in accordance with the requirements of Rules for Safety and Health at Work in electrical installations of electrical and heating plants and electrical networks and general conditions of contracts for use of distribution networks of "CEZ Distribution Bulgaria", and other normative documents regulating the process.

All employees, performing the activities regarding the taking of data from commercial metering devices, finding and marking the technical faults and their introduction into portable terminals must have at least third qualifying group as required by the Rules for Safety and Health at Work in electrical installations of electrical and heating plants and electrical networks (PBZREUETTSEM).

All employees performing the work called. Otchetnitsi should wear name tag.

The stages of reporting are:

1. The reporting is done by Reporters with portable terminals;

2. Get information about Reporting units (regions accounting and reporting periods) to periods of reading or working areas that should be taken during the month. The information is supplied by the "CEZ Distribution Bulgaria" not later than the last four (4) working days;

3. Targeting units for the upcoming reporting period to the corresponding handheld terminals. Performed by the "CEZ Distribution Bulgaria" not later than the beginning of the business day on which should start the reporting unit;

4. The reading of meters from a specific period must be carried out within it, as the reporting period of a measuring point should not exceed 31 days from the 
previous report. The reporting of a reporting unit is considered to be completed after the reporting unit is enclosed in the terminal and made a successful synchronization with the server. If necessary, the Company specifies the reporting date of a meter;

5. Synchronization of the hand terminal before beginning work. Performed by the Reporter on receipt of the units for the account. There are two consecutive synchronizations performed - to obtain the unit of account and confirmation of receipt;

6. Locate and electric power meters;

7. Reporting meter readings and recording them in hand terminal;

8. Required recorded accurate readings of the meter;

9. Check the recorded testimony;

10.Inspection for damage and irregularities in the measurement group and introduction if necessary of appropriate code for the anomaly;

11.Submission of information by recording the relevant code, failure of components of the measurement group;

12. Submission of information by recording the corresponding code in nesveren in real time tariff switch, and other anomalies in the operation of tariff switch;

13. Submission of information on suspected misuse of power. Energy;

14. Submission of information when there is an inconsistency on the elements of the measurement group in the information system and the place of consumption;

15.Submission of information on the type of object (VGK value); Submission of information about the place of installation of power meters (VGK value);

16.In unsecured access to the commercial metering devices installed in the property to the user in manual terminal records relevant code and prominently displayed at the entrance of the site left a message containing information on the date and time of the visit;

\section{Paper reporting Using} Terminals
17.Performing statements of date and time agreed with the client - information is provided to the Contractor at least three days before the agreed date;

18. When visiting the site of where the meters are downloadable GPS location. If the meters are indoors GPS coordinates downloaded directly in front of the site;

19. All meters in a board marked with some GPS location;

20.In the establishment of "anomalies" in a preset list, a photo documenting is done of the commercial metering / JTI /, as in the picture (pictures) clearly visible testimony to all tariffs and serial number of the JTI; (pictures) clearly visible testimony to all tariffs and serial number of the JTI.

21.In establishing a big difference between data JTI terminal and actual data of the JTI recorded real data and fotodokumentira, like the picture The history of accounting for electricity is presented in Figure 1.

In the beginning, until 2008 the reporting was done by the use of carriage. The disadvantages are that there is a 100 percent commitment to the region and collector, $100 \%$ work on paper / manual data processing / handling and more than 9,000 booklets. Also there are other significant shortcomings in the way the paper reporting such as:

- Low level of protection of archives with primary information;

- Low level of accessibility to primary data;

- Lack of controls at the time of reporting;

- Multiple errors in reporting and data entry;

- Low level of data organization JTI and places of consumption;

- Lack of opportunities for uniquely identifying the location of the site;

- Lack of opportunity to refine the information by introducing additional data on the accounts; - Lack of opportunity for systematic collection of data objects.

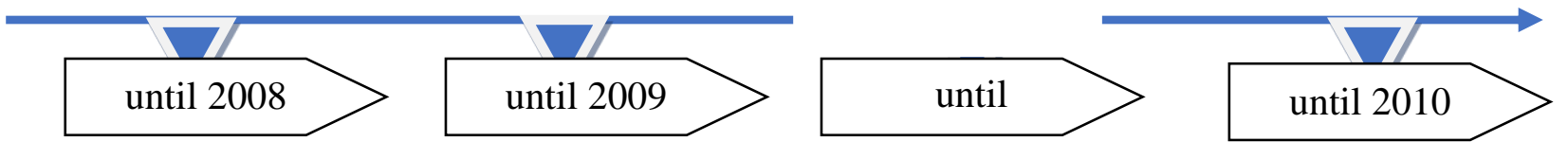

Figure 1. History of the measurement of electricity 
At present, the measurement from spent energy, from a place of consumption of electricity is done by installed individual commercial metering devices. Quantities consumed electric energy accumulated in the internal registers of the commercial metering devices and displayed on an electronic display or affect the mechanical counting mechanism by accumulation of numeric values, called testimony.

Commercial metering devices come in two main types - mechanical (induction) and static (electronic). The readings of the commercial metering devices became generally in two ways - on site or remotely.

Giving evidence of mechanical commercial metering is done entirely through site visits, while static - depending on their functional capabilities, supplied with additional components, modules or software giving evidence is done locally or remotely.

Each method of accounting has its advantages and disadvantages, some of the main advantages and disadvantages are:

1. The taking of evidence by a site visit

a) benefits:

- Timely identification of damaged elements of the metering unit that can be removed in a short time and not bring harm to the client or company;

- Timely identification of unauthorized encroachment on the commercial measurement (or other unauthorized manipulation) to disregard for the consumed amount of electricity;

- Timely establishment of damage related to the safe operation of facilities (so avoiding or at least reducing the risk of occurrence of accidents).

b) shortcomings:

- Admission of subjective errors;

- Lack of easy processing capacity of raw data;

- Costs related to personnel and transport;

- Strong dependence on weather conditions and the specificity of a given place of consumption (availability of access, availability of roads, addresses, etc.).

Remote readings

a) benefits:

- Availability of easily accessible for inspections and analyzes database;

- Lack of mistakes;

- Reduced personnel costs and transport;
- Availability of information from the commercial measurement in real or near real time;

- Reduced dependence on weather conditions and problems at the place of consumption;

b) shortcomings:

- High value commercial metering devices and the necessary additional equipment and software;

- Strongly dependent on the quality of the network and available interference therein or by the presence and the quality of the GSM connection for data transmission;

- Lack of opportunity for the timely establishment of various anomalies and unauthorized access.

Places of consumption located throughout the serviced territory are geographically divided into small separate groups. Data on places of consumption and commercial metering devices included in these groups are entered in units (TIR). The reporting entity is a paper in which they entered the necessary data for each measurement point, the client and the commercial measurement. All units are grouped into reporting periods, which ape evenly distributed throughout the month.

They are reported cyclically within the relevant period. Reporting is done by Reporters, each of which is informed of the measured units, which they must be measure throughou that period.

Before the start of every measuring, every accountant gets Carnets, which must be taken into account. Within the measuring period and the visited places of consumption, they have to enter data reported in Carnets, if necessary, enter various notes related to specific reports or the places of consumption, and enter information about anomalies, etc.

After completing the measurements, the results are being transported to processing centers where the data is manually entered in the customer information system. After entering the data, reporting agents will return to the places they've made the measurements and wait until the next period in which a carnet must be measured.

The whole process of reporting manually entering data in the TIR and client system, transportation between centers for reporting and processing centers is associated with a number of deficiencies and serious difficulties. 
The problems stem mainly from the fact that they use paper for entering the relevant data. Shortcomings identified are:

- A major opportunity for error in recording of evidence on paper (without lack of any independent accounting officer controls);

- Lack of ability to control the reporting process;

- Probability of error in manually entering data in the customer information system; - The need for TIR transport between centers for reporting and processing;

- Lack of a unified database containing information from primary reports;

- An extremely difficult process for entering and maintaining updated data on places of consumption,

- The places of consumption, regardless of administrative addresses.

\section{CONCLUSION}

To avoid disadvantages in taking the data from the commercial measurement and recording them on paper, there are two possible ways forward:

\section{Switching to remote measurement of the commercial metering devices}

It is necessary to purchase a suitable commercial measuring devices to replace existing networks, so that there is minimal interference to prevent the process of data transmission. In this case all of the advantages are lost of the readings of the commercial metering devices in place and appear all the shortcomings of remote taking of evidence.

Furthermore, in this way of avoiding problems that may arise from the use of paper is accompanied by the following disadvantages:

- $\quad$ very high investment for the purchase of commercial metering devices;

- Additional investment for the reconstruction of existing power networks;

- $\quad$ The need for a long time to perform the process;

- The inability of return of investment in customers with less consumption of electricity;

- Maintaining a pool of costly commercial metering devices;

- $\quad$ Lack of opportunity to use dismantled fit commercial metering devices.

As advantages, it can be pointed out the measurements of details remotely.

Considering all these facts switching to remote reading, it is effective in very large customers, where there are legal requirements that can not be met by "ordinary" commercial metering devices to places of consumption, where the investment is paid by the large amount of consumed electricity or risky places of consumption (very remote risk clients, etc.).

The second path of development is:

\section{Replace the paper carrier (carneta) with handheld portable terminals}

In this case, all benefits from taking the measurements statements from the commercial measurements, are visiting on the site, at the same time avoid or reduce many of the disadvantages of this type of activity.

Hand-held portable terminals are modern electronic devices equipped with an operating system, software for securing business, GPS agent to capture GPS coordinates navigation software, bar code reader, photo camera for making pictures.

Each speacialist is equipped with a personal terminal,so that the operation can go smoother, and he is performed precise control (always know which terminal, respectively, which takes into account, introduced an indication).

In the customer information system all the places of consumption are grouped into units, which are analogues of paper carnet. There is special software, server application which connects the customer information system and terminals. There are stores and primary and unchangeable database.

This database, just like paper card contains all the information in the form that was introduced by reporting, such data can not be changed. Unlike paper TIR this information is generally available (for those who have the appropriate access rights), regardless of where the user is also given a large number of reports and statements.

Each terminal has the application software installed, through which it operates on the readings of the commercial metering devices.

Before the beginning of each measuring perriod, orders are being created to account for the commercial metering devices, which are provided for measuring during the relevant period. E. units are transferred to the server application software managing the process of reporting terminals. Each measuring unit contains current information at the time of creation of orders for the place of consumption (address, type of facility, location of installation of the device for commercial metering, GPS coordinates, expected tolerance 
of consumption, other information supporting the process of reporting) customer for the commercial metering devices (serial number, type, date of the previous report, previous indication).

Before starting work the electronic units are directed from the server to the terminals of reporting. The connection is done via GPRS data transmission, i.e. does not require connection cable between the computer and the terminal to implement the transfer. This significantly facilitates the work and reduces transportation costs.

The person responsible for the measurements goes to the place of consumption and captures readings of the commercial metering devices and inputs the data into the terminal. If entries are outside the expected tolerance of consumption of a given measurement point, the terminal beeps and requires confirmation of the veracity of the report.

The expected tolerance of consumption is automatically determined by the customer information system based on historical consumption data given place of consumption. Record the seasonality previous consumption data and similar to last year. This comparison of actual and expected consumption significantly reduces the occurrence of errors of a subjective nature.

During the measurements, the responsible agent inspects the entire measurement group and submits information by writing the appropriate codes and notes in the terminal. There is a possibility, if necessary, to make pictures of the place.

The reporting agent makes a connection to the server (synchronization) where all new data is being transferred and then goes other terminal units (if targeted ones). From there, the data is automatically transferred to the customer information system, which remains to be stored on a server applicatio, servicing the terminals.

In the customer information system the data is being checked, analyzed for any errors, taking into account the recorded remarks, the pictures, the history of consumption of the respective place of consumption, etc. If it becomes necessary, it can be rechecked in place. After checking the data, they are ready for invoicing. Recorded information on anomalies is drawn from units responsible for taking action to remedy them.
In each report is done automatically capture GPS location. This helps both significantly greater control and navigation to the site next reporting or other activity (replacement device for commercial metering, removal of an accident, etc.).

The availability of accurate GPS location of the places of consumption, released the strong commitment of the measuring agent with the administrative addresses, their availability and fidelity.

The reporting system ensures that the data of the meters can not be manipulated. Electrometers are reported every month, and to reduce uncertainties as a result of human error, within three months, three different officials reported the same electrometer so admission of subjective errors is minimized. In some distribution companies as "CEZ Distribution Bulgaria" JSC has established a reporting system, which ensures that they cannot manipulate the data of the measuring devices.

Hand-held portable terminals make a beep sound and want confirmation of input readings when they are outside of the expected tolerance system that is individual for each measurement point and depends on the history of consumption. When entering each statement, the terminal automatically records GPS coordinates, and allows capturing.

Thus steps have been taken electric energy consumption to be read correctly, and readings from the meters to be reflected accurately in the monthly invoices.

Meters that are installed on the network, comply with all requirements of the Bulgarian legislation.

In each invoice CEZ indicate to customers during the next counting, so that consumers are informed on a monthly basis for the length of the upcoming reporting period.

As a conclusion, it can be said that the most suitable option for replacement of reporting on paper is reporting using portable terminals. Transition is associated with less investment compared with remote sensing, faster time to implement, to retain the advantages of visiting the place of consumption, while part of the drawbacks are avoided through the use of modern technologies. 


\section{REFERENCES}

1. Rules for measuring the quantity of electricity, 2013.

2. Energy Law, N07/09.12.2003.

3. Ordinance on measuring instruments subject to metrological control, N 239/24.10.2003.
4. Ivanova D., Diversification of production, University publishing house, Plovdiv, ISBN 978-954-423-884-1, 2013.

5. Stoilov D., Analysis of the electricity market in Bulgaria, Technical University Sofia, 2013.

6. www.cez.bg 
